\title{
Nível Tecnológico e Rentabilidade de Produção de Mel de Abelha (Apis Mellifera) no Ceará
}

\author{
Débora Gaspar Feitosa Freitas ${ }^{1}$ \\ Ahmad Saeed Khan² \\ Lúcia Maria Ramos Silva ${ }^{3}$
}

Resumo: O presente estudo procurou analisar a apicultura no Estado do Ceará, enfocando a produção de mel de abelhas (Apis mellifera) nos principais municípios produtores do Estado, mais precisamente fazendo uma análise do nível tecnológico empregado na produção, bem como avaliando a rentabilidade da atividade. A pesquisa se realizou através de coleta de dados primários por meio de entrevistas diretas com os produtores nos municípios de Mombaça, Pacajús e Chorozinho, no mês de outubro de 2002. Para avaliação do nível tecnológico, dividiu-se o sistema de produção de mel em cinco componentes: uso de equipamentos, manejo, colheita, pós-colheita e gestão; daí foram desenvolvidos índices tecnológicos para cada um separadamente e para o conjunto deles, com base na respectiva tecnologia recomendada, sendo que, quanto mais próximo da tecnologia recomendada, maior é o valor deste índice e, portanto, melhor o nível tecnológico. A avaliação da rentabilidade foi feita utilizando-se a metodologia do Sistema Integrado de Custos Agropecuários - CUSTAGRI. Os principais resultados obtidos

\footnotetext{
${ }^{1}$ Economista, MS, Doutoranda em Economia - Universidade Federal do Ceará. E-mail: deboragas@ig.com.br

${ }^{2}$ Eng. Agrônomo, Ph.D. em Economia Agrícola e Recursos Naturais. Professor Titular do Departamento de Economia Agrícola da Universidade Federal do Ceará. Bolsista do CNPq. E-mail: saeed@ufc.br

${ }^{3}$ Eng. Agrônoma, Livre Docência, Professora Adjunta IV do Departamento de Economia Agrícola da Universidade Federal do Ceará.E-mail: silvalramos@ufc.br
} 
mostram que o nível tecnológico dos produtores de mel é considerado bom, sendo que na pós-colheita apresenta melhores índices, enquanto na gestão foram encontrados os mais baixos índices. No que se refere à rentabilidade, a produção de mel é uma atividade muito rentável, envolvendo baixos custos e podendo chegar a elevados índices de lucratividade.

Palavras-chave: Apicultura, nível tecnológico, rentabilidade, Ceará.

\section{1 - Introdução}

A apicultura é uma atividade de grande importância, pois apresenta uma alternativa de ocupação e renda para o homem do campo. É uma atividade de fácil manutenção e de baixo custo inicial em relação às demais atividades agropecuárias.

Esta atividade desperta muito interesse em diversos segmentos da sociedade por se tratar de uma atividade que corresponde ao tripé da sustentabilidade: o social, o econômico e o ambiental. O social por se tratar de uma forma de geração de ocupação e emprego no campo. Quanto ao fator econômico, além da geração de renda, há a possibilidade de obtenção de bons lucros, e na questão ambiental pelo fato de as abelhas atuarem como polinizadores naturais de espécies nativas e cultivadas, preservando-as e conseqüentemente contribuindo para o equilíbrio do ecossistema e manutenção da biodiversidade (PAXTON, 1995).

Os principais produtos obtidos e comercializados da atividade apícola são o mel, a cera, a própolis, a geléia real e o veneno (apitoxina). Há também um segmento da apicultura que vem se desenvolvendo ao longo dos últimos anos, que é o de serviços de polinização, em que as colméias são alugadas para produtores de outra cultura agrícola com a finalidade de aumento da produção desta cultura (FREITAS, 1998).

O mel é considerado o produto apícola mais fácil de ser explorado, sendo também o mais conhecido e aquele com maiores possibilidades de comercialização. Além de ser um alimento, é também utilizado em indústrias farmacêuticas e cosméticas, pelas suas conhecidas ações terapêuticas.

O Estado do Ceará aparece nas estatísticas do IBGE como o segundo 
maior produtor de mel de abelhas da região Nordeste, participando com cerca de $25 \%$ da produção, e ainda com grande potencial de crescimento, ficando atrás do Estado do Piauí que detém quase $50 \%$ da produção na região nordestina.

Além da maior demanda e pelos bons preços alcançados pelo produto no ano de 2001, a atividade desperta grande interesse por se tratar de uma atividade que não exige muito tempo, nem requer muita sofisticação em termos tecnológicos. Apesar de existirem inovações de equipamentos e técnicas - que sem dúvida ajudam bastante na melhoria da atividade -, a produtividade na apicultura está relacionada principalmente ao manejo adequado e às condições da flora apícola que, adicionada às novas técnicas e à eficiência na comercialização, fazem-na destacar-se dentre as atividades agropecuárias.

Segundo VILELA (2000), seguindo-se a tecnologia recomendada na produção e comercializando o mel de maneira adequada, espera-se alta rentabilidade na atividade principalmente se comparada aos demais negócios agropecuários.

Tendo em vista a importância e a potencialidade da apicultura no Estado, considera-se importante a realização de estudos que permitam conhecer e avaliar o nível tecnológico dos produtores de mel nos principais municípios produtores no Estado do Ceará e a rentabilidade da atividade.

\section{2 - Aspectos Conceituais}

Na Teoria do Desenvolvimento Econômico, SCHUMPETER defende a tecnologia como elemento essencial da dinâmica capitalista, e analisa o processo de transformação que essa economia aufere quando se introduz uma inovação tecnológica radical em seu processo de produção (SILVA, 1995). O autor declara que a tecnologia é a responsável por mudanças no comportamento dos agentes econômicos, realocação de recursos, destruição dos métodos tradicionais de produção e mudança qualitativa na estrutura econômica.

Entre essas mudanças, Schumpeter mencionou que a inovação tecnológica é um fenômeno puramente econômico da história do capitalismo, e elaborou a teoria da inovação, que em linhas gerais defende 
que, para que esta transforme o sistema econômico, é necessário que os empreendedores surjam em blocos e não distribuídos de maneira uniforme ao longo do tempo; revela ainda que o êxito do empreendimento é que induzirá o ingresso de outros empreendedores, difundindo, assim, a inovação, o que caracterizou a divisão da teoria em três etapas: invenção, inovação e difusão.

Já a Teoria Neoclássica não se aprofundou nos assuntos relacionados à tecnologia até meados da década de 1950, quando os autores em seus modelos de crescimento econômico enfatizavam a terra, capital e trabalho, e, apesar de reconhecer o progresso tecnológico, este não era incluído formalmente no modelo. HICKS, citado por SOUZA (2000), tratou da inovação tecnológica em relação ao trabalho, acreditando que não haveria razão para achar que as inovações fossem por elas mesmas poupadoras de trabalho, mas que os empresários tenderiam a buscar inovações que lhes poupassem mão-de-obra para compensar aumentos nos seus custos; também formulou uma teoria em que as inovações eram consideradas como induzidas pela escassez relativa dos fatores de produção.

Nos trabalhos de Schumpeter e Hicks, destacaram-se importantes conceitos, como inovação transformadora da estrutura produtiva e inovação induzida, caracterizando os chamados modelos pontuais. De igual importância foram os modelos de economia dual, que colocaram a modernização do setor agrícola, através da adoção de inovações tecnológicas, como condição necessária ao desenvolvimento da economia.

A adoção de novas tecnologias pode elevar os níveis de produtividade de uma empresa, seja ela agrícola ou não, beneficiando positivamente a economia. Embora as novas tecnologias sejam de conhecimento dos produtores, nem todos a adotam, muitas vezes por fatores socioeconômicos relacionados (KHAN et all, 1991).

Nos últimos anos, a revolução tecnocientífica ocorrida principalmente no ramo das Telecomunicações e Informática, Química e Genética, revolucionou todos os ramos da Economia, principalmente a atividade agrícola (MIRANDA, 2001).

A atividade agrícola também foi alvo de impactos diante da incorporação de inovações mecânicas, químicas e genéticas na sua base produtiva. Essas inovações refletiam-se nas empresas, onde foram obser- 
vadas a substituição de equipamentos e incorporação de novos modos de administração de trabalho e organização da produção.

De acordo com MIRANDA (2001), há também uma linha relativamente nova de pensamento que enfoca que as empresas em geral contam com um processo inovativo em tecnologia, quando implementam ações estratégicas, expressando a incorporação tecnológica em termos de eficiência produtiva, diversificação de produtos, gestão, controle de qualidade e planejamento estratégico.

Há ainda uma linha de pesquisa que trata da capacidade das empresas em se apropriar de avanços tecnológicos como forma de estratégia de competitividade nos mercados, considerando que a constante inovação tecnológica em uma empresa determina a criação ou manutenção da competitividade desta numa região.

\section{3 - Metodologia}

\section{1 - Área Geográfica de Estudo}

Foram escolhidos os municípios de Pacajus, Chorozinho e Mombaça para o desenvolvimento da pesquisa, porque são municípios que apresentaram, de acordo com dados obtidos na Secretaria de Desenvolvimento Rural (SDR) 2002, nos últimos anos, uma produção representativa no setor apícola, destacando-se entre os dez municípios do Estado do Ceará com maior produção de mel.

\section{2 - Levantamento dos Dados}

Foram utilizados dados primários obtidos através de entrevistas diretas com os produtores nos municípios mencionados, no mês de outubro de 2002.

As informações referentes à tecnologia recomendada foram obtidas junto ao Departamento de Zootecnia, Setor de Apicultura, da Universidade Federal do Ceará (UFC).

Foram utilizados dados bibliográficos disponíveis em instituições de ensino e pesquisa, bem como dados estatísticos secundários encontrados em órgãos especializados como: Instituto Brasileiro de Geografia e 
Estatística (IBGE), Instituto de Planejamento do Ceará (IPLANCE), Empresa de Assistência Técnica e Extensão Rural do Ceará (EMATERCE), Secretaria de Desenvolvimento Rural (SDR), dentre outras.

\section{3 - Tamanho da Amostra}

Foi utilizada uma amostragem aleatória simples, cuja determinação do tamanho seguirá o método para amostras de populações finitas, conforme FONSECA e MARTINS (1996) :

$$
\mathrm{n}=\frac{z^{2} \cdot p \cdot q \cdot N}{d^{2} \cdot(N-1)+z^{2} \cdot p \cdot q}
$$

onde:

$\mathrm{n}=$ tamanho da amostra para populações finitas

$z^{2}=$ abscissa da normal padrão

$\mathrm{p}$ = estimativa da proporção da característica pesquisada no universo

$\mathrm{q}=1-\mathrm{p}$

$\mathrm{N}=$ tamanho da população

$\mathrm{d}=$ erro amostral

Assim, de acordo com o cálculo da amostra em função do tamanho da população em estudo, foram entrevistados 33 produtores de mel de abelhas no município de Mombaça, e nove produtores dos municípios de Pacajus e Chorozinho, tomados aleatoriamente.

\section{4 - Definição e Operacionalização das Variáveis}

\subsection{1 - Análise da Tecnologia}

De acordo com informações obtidas junto ao Departamento de Zootecnia da Universidade Federal do Ceará, e seguindo as referências de vários autores na área de apicultura, como WIESE (1985), COUTO \& COUTO (2002), FREE (1982), FREITAS (1998), pode-se descrever a tecnologia recomendada para a produção de mel.

Para a identificação do nível tecnológico, serão considerados os 
seguintes componentes do sistema de produção na apicultura: 1) Uso de Equipamentos; 2) Manejo; 3) Coleta e Processamento de Mel; 4) Pós-colheita de Mel e 5) Gestão.

As variáveis incluídas em cada tecnologia são apresentadas nas TABELAS 1A a 5A.

\section{5 - Métodos de Análise}

\subsection{1 - Determinação do Nível Tecnológico}

Para proceder a uma análise quantitativa dos diferenciais tecnológicos, é considerado cada um dos itens descritos anteriormente no sistema de produção na apicultura.

$\mathrm{Na}$ avaliação do nível tecnológico, é determinado inicialmente um índice tecnológico para cada produtor em cada um dos componentes que formarão o referido nível, conforme MIRANDA, 2001:

$I n_{j}=\sum_{i=y}^{m} \frac{a_{i}}{w_{n}}$

sendo, $w_{n}=\operatorname{Max} \sum_{i=y}^{m} a_{i}$ e dessa forma, $0 \leq \operatorname{In} n_{j} \leq 1$,

onde:

$I n_{j}=$ Índice de cada Tecnologia $n$ do produtor $j$;

$i=$ Variáveis utilizadas;

$n=$ Tecnologia utilizada;

$[y, m]=$ variáveis dentro do segmento $i$ referentes à tecnologia $n$; $a_{i}=$ representa o valor da adoção do elemento $\mathrm{x}_{i}$ da tecnologia $n$;

Assim, $\frac{a_{i}}{w_{n}}$ representa o peso de cada elemento $\mathrm{x}_{i}$ na constituição do índice tecnológico específico $n$, e

para a tecnologia de equipamentos, $\mathrm{n}=1, \mathrm{i}=[1 ; 15]$ e $\mathrm{w}_{1}=22$;

para a tecnologia de manejo, $\quad \mathrm{n}=2, \mathrm{i}=[16 ; 28]$ e $\mathrm{w}_{2}=21$;

para a tecnologia de colheita, $\quad \mathrm{n}=3, \mathrm{i}=[29 ; 34]$ e $\mathrm{w}_{3}=9$;

para a tecnologia de pós-colheita, $\quad \mathrm{n}=4, \mathrm{i}=[35 ; 38]$ e $\quad \mathrm{w}_{4}=7$;

para a tecnologia da gestão, $\quad \mathrm{n}=5, \mathrm{i}=[39 ; 46]$ e $\quad \mathrm{w}_{5}=8$. 
O índice tecnológico médio específico para o conjunto de produtores é dado pelo somatório dos índices específicos dos produtores individuais dividido pelo número de produtores entrevistados, demonstrado pela equação:

$I T_{n}=\frac{1}{\mathrm{z}} \sum_{\mathrm{j}=1}^{\mathrm{z}} \ln \mathrm{j}$

onde:

$\mathrm{j}=$ Número de produtores (variando de $1 \mathrm{a} \mathrm{z}$ )

$n=$ Tecnologia utilizada

O índice tecnológico geral de um produtor, incluindo-se todas as tecnologias, pode ser obtido da seguinte forma:

$I P_{j}=\frac{1}{5} \sum_{1}^{5} \ln _{\mathrm{j}}$

Assim, o índice tecnológico da produção de mel na área de estudo, considerando-se todos os produtores, será expresso como a seguir:

$I G=\frac{1}{\mathrm{j}} \sum_{1}^{j} I P_{j}$

Com base nos valores obtidos dos índices (que variam de zero a um), determina-se o nível tecnológico dos produtores de mel, considerando-se que quanto mais próximo do valor máximo (um), melhor será o nível tecnológico dos respectivos produtores.

\subsection{2 - Indicadores de Rentabilidade}

A determinação dos custos e dos indicadores de rentabilidade que serão utilizados nesta pesquisa tem por base os conceitos utilizados por MARTIN et al. (1998) no desenvolvimento do Sistema Integrado de Custos Agropecuários (CUSTAGRI), pesquisa feita com a cooperação entre o Instituto de Economia Aplicada (IEA) em parceria com o Centro Nacional de Pesquisa Tecnológica em Informática para a Agricultura (CNPTIA - EMBRAPA). 


\section{4 - Resultados e Discussão}

\section{1 - Índice Tecnológico Geral da Produção de Mel}

Na composição do nível tecnológico, determinou-se um índice geral composto de índices referentes a cada tecnologia e posteriormente suas participações relativas no índice tecnológico geral da produção de mel. Conforme mencionado, definiu-se :

IG, o índice que avalia o nível tecnológico geral da produção de mel, englobando todas as tecnologias: uso de equipamentos, manejo, colheita, pós-colheita e gestão.

4.1.1 - Índice tecnológico referente à produção de mel, incluindo as tecnologias de uso de equipamentos, manejo, colheita, pós-colheita e gestão (IG)

Conforme se observa na TABELA 1, o nível tecnológico geral dos apicultores em Mombaça foi dentro do padrão tecnológico $\mathrm{B}$, (IG = 0,592). Ainda se encontrou $9,09 \%$ dos apicultores no padrão $C$ e $6,06 \%$ no padrão A, sendo, portanto, a maioria inserida no padrão $B$.

Nos municípios de Pacajus e Chorozinho, o índice foi superior ao do município de Mombaça, ficando em $I G=0,675$, no entanto enquadra-se no padrão tecnológico $B$, que ficou representado por $88,89 \%$ dos entrevistados, enquanto $11,11 \%$ situaram-se no padrão A. 
Tabela 1 - Contribuição das tecnologias de uso de equipamentos, manejo, colheita, pós-colheita e gestão na composição do índice geral (IG).

\begin{tabular}{|c|c|c|c|c|c|c|}
\hline \multirow{3}{*}{ Especificação } & \multicolumn{4}{|c|}{ Municípios } & \multirow{2}{*}{\multicolumn{2}{|c|}{ Amostra Total }} \\
\hline & \multicolumn{2}{|c|}{ Mombaça } & \multicolumn{2}{|c|}{ Pacajus e Chorozinho } & & \\
\hline & Absoluta & $\%$ & Absoluta & $\%$ & Absoluta & $\%$ \\
\hline Equipamentos & 0,1394 & 23,52 & 0,1354 & 20,04 & 0,1385 & 22,69 \\
\hline Manejo & 0,1164 & 19,64 & 0,1365 & 20,20 & 0,1206 & 19,76 \\
\hline Colheita & 0,1132 & 19,10 & 0,1432 & 21,19 & 0,1196 & 19,60 \\
\hline Pós-colheita & 0,1524 & 25,72 & 0,1524 & 22,55 & 0,1524 & 24,97 \\
\hline Gestão & 0,0712 & 12,02 & 0,1083 & 16,02 & 0,0792 & 12,98 \\
\hline IG & 0,5926 & 100,00 & 0,6758 & 100,00 & 0,6103 & 100,00 \\
\hline Menor Indicador & 0,4907 & - & 0,5532 & - & 0,4907 & 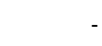 \\
\hline Maior Indicador & 0,7875 & - & 0,8407 & - & 0,8407 & - \\
\hline
\end{tabular}

Fonte: Dados da pesquisa.

Para os três municípios juntos, o índice tecnológico geral da produção de mel foi de IG $=0,610$, ou seja, o nível tecnológico foi compatível com o padrão B, podendo ser considerado bom, em razão dos produtores adotarem mais de $60,00 \%$ da tecnologia recomendada.

4.1.2 - Contribuição de cada tecnologia na formação do índice IG

No município de Mombaça, observa-se na Tabela 1 que o indicador que tem maior participação na composição do índice é a pós-colheita, que contribui com $25,72 \%$ na formação deste; já o indicador gestão é novamente o que apresenta menor contribuição: apenas 12,02\% .

Nos municípios de Pacajus e Chorozinho, semelhante ao que acontece no município de Mombaça, a maior participação é da tecnologia de pós-colheita, contribuindo com $22,55 \%$ na composição do índice, embora os outros índices tenham valores percentuais de participação bem aproximados. A tecnologia da gestão apresenta menor valor, tanto em valores absolutos como relativos, sendo um pouco mais elevado do que no município de Mombaça, conforme se observa na Tabela 1.

Para a amostra total, as contribuições das tecnologias seguem o mesmo comportamento dos municípios individualmente, onde a póscolheita apresenta maior participação e a gestão a menor. 


\section{2 - Determinação dos Indicadores de Rentabilidade}

A TABELA 2 apresenta o sumário dos indicadores de rentabilidade da produção de mel, para os municípios em análise.

Tabela 2 - Indicadores de rentabilidade da produção de $100 \mathrm{~kg}$ de mel de abelhas (Apis mellífera) - 2002.

\begin{tabular}{l|r|r|r}
\hline \multirow{2}{*}{ Item } & \multicolumn{3}{c}{ Valor Total } \\
\cline { 2 - 4 } & Mombaça & Pacajus e Chorozinho & Amostra Total \\
\hline Lucro Operacional (R\$/100 kg) & 142,04 & 259,12 & 167,13 \\
Receita Bruta & 257,82 & 396,89 & 287,62 \\
Custo Operacional Total & 115,78 & 137,77 & 120,49 \\
Índice de Lucratividade (\%) & 54,47 & 65,39 & 56,81 \\
Lucro operacional & 142,04 & 259,12 & 167,13 \\
Receita Bruta & 257,82 & 396,89 & 287,62 \\
MBCOE (\%) & $1.072,63$ & 725,50 & 998,24 \\
Receita Bruta & 257,82 & 396,89 & 287,62 \\
COE & 32,55 & 68,85 & 40,33 \\
MBC0T (\%) & 168,74 & 206,77 & 176,89 \\
Receita Bruta & 257,82 & 396,89 & 287,62 \\
COT & 115,78 & 137,77 & 120,49 \\
PNCOE (kg) & 12,62 & 17,34 & 14,00 \\
COE & 32,55 & 68,85 & 40,33 \\
Preço do Mel (kg) & 2,58 & 3,97 & 2,88 \\
PNCOT (kg) & 44,88 & 34,70 & 41,84 \\
COT & 115,78 & 137,77 & 120,49 \\
Preço do Mel (kg) & 2,58 & 3,97 & 2,88 \\
\hline
\end{tabular}

Fonte: Dados da pesquisa

O Lucro Operacional (LO), que corresponde à diferença entre a Receita Bruta (RB) e o Custo Operacional Total (COT), para uma quantidade produzida de $100 \mathrm{~kg}$, no caso do município de Mombaça correspondeu a R $\$ 142,04$, e nos municípios de Pacajus e Chorozinho o valor correspondente foi de $\mathrm{R} \$ 259,12$.

Os índices de lucratividade (IL) foram, respectivamente, $54,47 \%$ e 65,39\%, nos municípios de Mombaça e Pacajus e Chorozinho. No caso da amostra total, o LO obteve o valor de R\$167,13, e o IL foi de $56,81 \%$. Tais valores mostram que a apicultura é uma atividade extremamente rentável e que proporcionou alta lucratividade no ano 
em estudo. É importante ressaltar que os dados coletados se referiram apenas ao ano de 2002, e que por fatores externos o mel alcançou um preço mais elevado do que os preços esperados, ademais, como referido, os custos de oportunidade dos fatores de produção não foram considerados.

Na Tabela 2, tem-se o sumário das Margens Brutas e Ponto de Nivelamento calculados. No município de Mombaça, a Margem Bruta em relação ao custo operacional total (MBCOT) é de 168,74\%, o que indica que após pagarem-se os custos operacionais totais, os produtores de mel dispõem ainda de $168,74 \%$ sobre o valor destes custos, ou melhor, de acordo com a definição da Margem Bruta, esse percentual indica que os produtores dispõem de uma quantidade de recursos $168,74 \%$ superior ao COT, após pagarem-se todos estes custos. Este montante poderia ser utilizado para cobrir a remuneração ao capital, a terra e à capacidade empresarial do proprietário. No caso dos produtores do município de Pacajus e Chorozinho, essa margem é de 206,77\%, e para a amostra total a MBCOT é de $176,89 \%$.

O Ponto de Nivelamento em relação ao COE (PNCOE) no município de Mombaça é de 17,34 kg e nos municípios de Pacajus e Chorozinho é de 12,62 kg. Já para a amostra total, o PNCOE é de $14 \mathrm{~kg}$. Esses resultados significam que são necessários menos de $18 \mathrm{~kg}$ de mel numa produção de $100 \mathrm{~kg}$, para se pagarem os custos operacionais efetivos.

O Ponto de Nivelamento em relação ao COT (PNCOT) no município de Mombaça é de 44,88 kg, ou seja, para cada $100 \mathrm{~kg}$ de mel produzidos são necessários $44,88 \mathrm{~kg}$ para se cobrir os custos operacionais totais. Nos municípios de Pacajus e Chorozinho, o PNCOT é de 34,70 kg. Dessa forma, verifica-se que os Pontos de Nivelamento em Mombaça são maiores do que nos demais municípios, e, conseqüentemente, a produção necessária para se cobrir os custos é superior neste município aos demais. Para a amostra total, o PNCOT é de 41,84 kg , ou seja, em uma produção de $100 \mathrm{~kg}$, é necessário menos da metade da produção para se pagar os custos operacionais totais.

Estes resultados poderão servir de incentivos para aqueles que estão dispostos a investir nesta atividade. 


\section{5 - Conclusões}

O nível tecnológico dos produtores de mel é considerado bom. Nos municípios de Pacajus e Chorozinho, estes foram um pouco maiores do que no município de Mombaça, embora ficando todos no mesmo padrão B.

Nas tecnologias de uso de equipamentos, manejo e colheita, o nível tecnológico é considerado bom nos municípios analisados, pois situam-se no padrão B, ou seja, mais da metade da tecnologia recomendada é utilizada, embora haja variações nos índices encontrados, onde se pode encontrar produtores nos demais padrões, estes representam apenas pequena parcela de produtores.

A tecnologia de pós-colheita teve o mesmo valor nos municípios estudados $(0,7619)$ e apresenta melhor nível tecnológico entre as demais tecnologias, situando-se no padrão A. Já a tecnologia da gestão apresentou um baixo nível tecnológico no município de Mombaça, mas um bom nível em Pacajus e Chorozinho, o que pode estar associado a um maior nível de escolaridade nestes dois últimos municípios.

As tecnologias de pós-colheita e de uso de equipamentos apresentaram maior contribuição à formação do índice geral, enquanto que a tecnologia da gestão participa com a menor contribuição.

Os resultados demonstram que a produção de mel é uma atividade muito rentável, podendo chegar a altos índices de lucratividade, incorrendo em poucos custos. Nos municípios de Pacajus e Chorozinho a lucratividade é maior do que no município de Mombaça, e este fato pode ser atribuído aos melhores preços recebidos pelos apicultores dos primeiros municípios em razão da existência de um canal de comercialização direta e de sua localização próxima à capital do Estado, e também a maior produtividade obtida por estes apicultores. Entretanto, deve-se ressaltar que os custos operacionais são inferiores no município de Mombaça. De modo geral, a apicultura mostra-se como uma boa opção para geração de renda no campo. 


\section{6 - Referências Bibliográficas}

COUTO, R.H.N. e COUTO, L..A. Apicultura: manejo e produtos. 2 ed. Jaboticabal: FUNEP, 2002. 191p.

FONSECA, J.S. de; MARTINS, G. de A. Curso de estatística. 6 ed. São Paulo: Atlas, 1996. 320p.

FREE, J.B. Bees and mankind. London: George Allen \& Unwin (Publishers) Ltd. 1982. 155p.

FREITAS, B.M. O uso de programas racionais de polinização em áreas agrícolas. Mensagem doce. N.46, p.16-20, São Paulo: APACAME, 1998.

FREITAS, B.M. A Vida das abelhas. Fortaleza: UFC.Craveiro \& Craveiro, 1999. CD Rom.

KHAN, A.S.; RIBEIRO,D.G.L.; SILVA, L.M.R.; MESQUITA, T.C. Adoção de tecnologia na produção da cana-de-açúcar na região do Cariri, Ceará. Revista da SOBER (CD Rom), 2002.

MARTIN, N.B. et al. Sistema integrado de custos agropecuários - CUSTAGRI. Informações econômicas. São Paulo, v. 28, n. 1, p. 7-27, jan. 1998.

MIRANDA, E.A.de A. Inovações tecnológicas na viticultura do sub-médio São Francisco. 191f. Tese de Doutorado (Doutorado em Economia): PIMES / UFPE. Recife, 2001.

PAXTON, R. Conserving wild bees. Bee World. N.76, v.2, p.53-55. Inglaterra, 1995.

SILVA, C.R.L. Inovação tecnológica e distribuição de renda: impacto distributivo dos ganhos de produtividade da agricultura brasileira. São Paulo: IEA - Instituto de Economia Agrícola, 1995.

SOUSA, F.L.M. Estudo sobre o nível tecnológico da agricultura familiar no Ceará. 107p. Dissertação (Mestrado em Economia Rural) DEA/CCA/UFC, Fortaleza: 2000.

VILELA, S. L. de O. A importância das novas atividades agrícolas ante a globalização: a apicultura no Estado do Piauí. Teresina: Embrapa Meio-Norte, 2000. 228p.

WIESE, H. (Coord.). Nova Apicultura. Porto Alegre: Agropecuária, 1985. 493p. 


\section{Apêndices}

Tabela 1a - Variáveis relativas à tecnologia de uso de equipamentos

\begin{tabular}{|c|c|c|}
\hline \multirow{2}{*}{ Variáveis } & \multicolumn{2}{|c|}{ Valor } \\
\hline & Utiliza & Não Utiliza \\
\hline \multirow{3}{*}{$\begin{array}{l}\mathrm{X}_{1} \text { - Indumentárias: } \\
\text { - alguns itens } \\
\text { - todos os itens }\end{array}$} & & 0 \\
\hline & 1 & \\
\hline & 2 & \\
\hline \multirow{3}{*}{$\begin{array}{l}\mathrm{X}_{2} \text { - Fumigador: - com combustível } \\
\text { - com esterco de animal } \\
\text { - com prod. orig.vegetal }\end{array}$} & & 0 \\
\hline & 1 & \\
\hline & 2 & \\
\hline \multirow{2}{*}{$\begin{array}{l}\mathrm{X}_{3} \text { - Formão: - simples } \\
\text { - com saca quadros }\end{array}$} & 1 & 0 \\
\hline & 2 & \\
\hline \multirow{3}{*}{$\begin{array}{l}\mathrm{X}_{4^{-}} \text {Vassourinha: } \\
\text { - origem animal } \\
\text { - origem sintética }\end{array}$} & & 0 \\
\hline & 1 & \\
\hline & 2 & \\
\hline \multirow{3}{*}{$\begin{array}{l}\mathrm{X}_{5} \text { - Colméia Langstroth: } \\
\text { - fora do padrão } \\
\text { - dentro do padrão }\end{array}$} & & 0 \\
\hline & 1 & \\
\hline & 2 & \\
\hline \multirow{3}{*}{$\begin{array}{l}\mathrm{X}_{6} \text { - Equipamentos em aço inox: } \\
\text { - alguns dos equipamentos } \\
\text { - todos os equipamentos }\end{array}$} & & 0 \\
\hline & 1 & \\
\hline & 2 & \\
\hline \multirow{3}{*}{$\begin{array}{l}\mathrm{X}_{7} \text { - Centrífuga: } \\
\text { - manual } \\
\text { - elétrica }\end{array}$} & & 0 \\
\hline & 1 & \\
\hline & 2 & \\
\hline $\mathrm{X}_{8}$ - Decantador & 1 & 0 \\
\hline $\mathrm{X}_{9}-$ Mesa desoperculadora & 1 & 0 \\
\hline $\mathrm{X}_{10}-$ Peneiras & 1 & 0 \\
\hline $\mathrm{X}_{11}-$ Bombas $\mathrm{p} /$ elevação de mel & 1 & 0 \\
\hline $\mathrm{X}_{12}-$ Homogeneizador de mel & 1 & 0 \\
\hline $\mathrm{X}_{13}-$ Descristalizador de mel & 1 & 0 \\
\hline $\mathrm{X}_{14}-$ Tela excluidora de rainhas & 1 & 0 \\
\hline $\mathrm{X}_{15}$ - Carretilha para incrustação de cera & 1 & 0 \\
\hline
\end{tabular}


Tabela 2a - Variáveis relativas à tecnologia de manejo

\begin{tabular}{|c|c|c|}
\hline \multirow[b]{2}{*}{ Variáveis } & \multicolumn{2}{|c|}{ Valor } \\
\hline & Utiliza & Não Utiliza \\
\hline $\mathrm{X}_{16}-$ Alimentação estimulante & 1 & 0 \\
\hline \multirow{3}{*}{$\begin{array}{l}\mathrm{X}_{17} \text { - Troca de Rainhas: } \\
\text { - rainhas próprias } \\
\text { - rainhas compradas }\end{array}$} & & 0 \\
\hline & 1 & \\
\hline & 2 & \\
\hline \multirow{3}{*}{$\begin{array}{l}\mathrm{X}_{18} \text { - Troca de cera alveolada: } \\
\text { - troca de } 2 \text { em } 2 \text { anos } \\
\text { - troca todo ano }\end{array}$} & & 0 \\
\hline & 1 & \\
\hline & 2 & \\
\hline $\mathrm{X}_{19}$ - Controle de enxameação & 1 & 0 \\
\hline $\mathrm{X}_{20}-$ Divisão de enxames & 1 & 0 \\
\hline$X_{21}$ - Desobstrução do ninho & 1 & 0 \\
\hline \multirow{3}{*}{$\begin{array}{l}\mathrm{X}_{22}-\text { Abertura de espaço p/ armazenar } \\
\text { - mel (melgueiras): faz } \\
\text {-faz só quando colhe mel }\end{array}$} & & 0 \\
\hline & 1 & \\
\hline & 2 & \\
\hline \multirow{3}{*}{$\begin{array}{l}\mathrm{X}_{23} \text { - Reserva de alimento: } \\
\text { - deixa só no ninho } \\
\text { - deixa no ninho e melgueira } \\
\end{array}$} & & 0 \\
\hline & 1 & \\
\hline & 2 & \\
\hline \multirow{3}{*}{$\begin{array}{l}\mathrm{X}_{24} \text { - Combate às traças e formigas: } \\
\text { - combate um ou outro } \\
\text { - combate ambos }\end{array}$} & & 0 \\
\hline & 1 & \\
\hline & 2 & \\
\hline \multirow{3}{*}{$\begin{array}{l}\mathrm{X}_{25} \text { - Alimentação artificial: } \\
\text { - protéica ou energética } \\
\text { - ambas }\end{array}$} & & 0 \\
\hline & 1 & \\
\hline & 2 & \\
\hline $\mathrm{X}_{26}-$ Ventilação & 1 & 0 \\
\hline \multirow{3}{*}{$\begin{array}{l}\mathrm{X}_{27} \text { - Sombreamento: } \\
\text { - artificial } \\
\text { - natural }\end{array}$} & & 0 \\
\hline & 1 & \\
\hline & 2 & \\
\hline \multirow{4}{*}{$\begin{array}{l}\mathrm{X}_{28} \text { - Distância da água: } \\
\text { - maior do que } 500 \mathrm{~m} \\
\text { - entre } 100 \mathrm{~m} \text { e } 500 \mathrm{~m} \\
\text { - menos de } 100 \mathrm{~m}\end{array}$} & & \\
\hline & & 0 \\
\hline & 1 & \\
\hline & 2 & \\
\hline
\end{tabular}


Tabela 3a - Variáveis relativas à tecnologia de colheita de mel

\begin{tabular}{|c|c|c|}
\hline \multirow[t]{2}{*}{ Variáveis } & \multicolumn{2}{|c|}{ Valor } \\
\hline & Utiliza & Não Utiliza \\
\hline $\mathrm{X}_{29}-$ Fumaça & 1 & 0 \\
\hline $\mathrm{X}_{30}$ - Escape-abelha & 1 & 0 \\
\hline \multirow{4}{*}{$\begin{array}{l}\mathrm{X}_{31} \text { - Técnica de coleta: } \\
\text { - bate a melgueira } \\
\text { - chacoalha os favos } \\
\text { - usa a vassourinha }\end{array}$} & & \\
\hline & & 0 \\
\hline & 1 & \\
\hline & 2 & \\
\hline $\mathrm{X}_{32}-$ Garfo desoperculador & 1 & 0 \\
\hline \multirow{3}{*}{$\begin{array}{l}\mathrm{X}_{33} \text { - Casa do Mel: } \\
\text { - fora do padrão } \\
\text { - dentro do padrão }\end{array}$} & & 0 \\
\hline & 1 & \\
\hline & 2 & \\
\hline \multirow{4}{*}{$\begin{array}{l}X_{34} \text { - Transporte das melgueiras: } \\
\text { - na mão ou usando algum animal } \\
\text { - no carrinho de mão } \\
\text { - no veículo }\end{array}$} & & \\
\hline & & 0 \\
\hline & 1 & \\
\hline & 2 & \\
\hline
\end{tabular}

Tabela 4a - Variáveis relativas à tecnologia de pós-colheita

\begin{tabular}{|c|c|c|}
\hline \multirow[b]{2}{*}{ Variáveis } & \multicolumn{2}{|c|}{ Valor } \\
\hline & Utiliza & Não Utiliza \\
\hline \multirow{3}{*}{$\begin{array}{l}\mathrm{X}_{35} \text { - Equipamentos de higiene: } \\
\text { - algum dos itens } \\
\text { todos os itens }\end{array}$} & & 0 \\
\hline & 1 & \\
\hline & 2 & \\
\hline \multirow{4}{*}{$\begin{array}{l}\mathrm{X}_{36} \text { - Armazenagem: } \\
\text { - sem proteção } \\
\text { - protegido do calor ou umidade } \\
\text { - protegido de ambos }\end{array}$} & & \\
\hline & & 0 \\
\hline & 1 & \\
\hline & 2 & \\
\hline \multirow{3}{*}{$\begin{array}{l}\mathrm{X}_{37} \text { - Recipientes para armazenagem: } \\
\text { - fora do padrão } \\
\text { - nos padrões }\end{array}$} & & 0 \\
\hline & 1 & \\
\hline & 2 & \\
\hline \multirow{2}{*}{$\begin{array}{l}\mathrm{X}_{38} \text { - Fracionamento do mel: manual } \\
\text { - automático }\end{array}$} & & 0 \\
\hline & 1 & \\
\hline
\end{tabular}


Tabela 5a - Variáveis relativas à tecnologia da gestão

\begin{tabular}{l|c|c}
\hline \multirow{2}{*}{ Variáveis } & \multicolumn{2}{c}{ Valor } \\
\cline { 2 - 3 } & Utiliza & Não Utiliza \\
\hline $\mathrm{X}_{39}-$ Contrato de prestação de serviços & 1 & 0 \\
\hline $\mathrm{X}_{40}-$ Informações sobre tendências de mercado & 1 & 0 \\
\hline $\mathrm{X}_{41}-$ Treinamento de funcionários & 1 & 0 \\
\hline $\mathrm{X}_{42}-$ Controle de qualidade & 1 & 0 \\
\hline $\mathrm{X}_{43}-$ Parceria: pesquisa & 1 & 0 \\
\hline $\mathrm{X}_{44}-$ Parceria: comercialização & 1 & 0 \\
\hline $\mathrm{X}_{45}-$ Marketing & 1 & 0 \\
\hline $\mathrm{X}_{46}-$ Informática & 1 & 0 \\
\hline
\end{tabular}

Recebido em maio de 2003 e revisto em janeiro de 2004. 